\title{
Healthy and Asthmatic Sounds Classification using Fuzzy Clustering based Cascade Classifier Methods
}

\author{
Güneş Harman \\ Assistant Professor \\ Department of Computer Engineering \\ Yalova University, Türkiye, Yalova
}

\begin{abstract}
In this study, our aim is to presents different cascade classifier models based on Fuzzy C-means clustering methods, to classified normal and asthmatic lung sounds. The lung sounds recorded from both left and right lobe of the lung and divided inspiration and expiration phases. The frequency spectrum is obtained by using Fast Fourier Transform (FFT). After obtaining the spectrums of healthy and asthmatic lung sounds, fuzzy c-mean is applied to cluster the spectrum and then supervised classifier ANN, Naive Bayes and SVM are applied to classified healthy and asthmatic lung sounds.
\end{abstract}

\section{General Terms}

Machine Learning.

\section{Keywords}

Lung sounds, Fuzzy c Means, ANN, Naïve Bayes, SVM.

\section{INTRODUCTION}

Breathing is vital to maintaining the function of life for why because every living cell within the human body requires a fresh supply of oxygen and removal of carbon dioxide in order to survive. The lungs play an important role in the complex system, which allows respiration to occur. The other significance is that, the sounds that come out of this organ's work used by the physician decide about abnormalities and the evaluation of lung disorders. Also, for another researcher whose study is about lung sound analysis or which is parallel to this issue.

On the bases of this information lung sounds comprise a fundamental clue about respiratory status. Lung sound is one of the most essential clues for differentiating of respiratory disease. Particular lung sounds characterized by the features of duration, intensity, pitch, and timing. The sounds are divided two major categories a base on acoustic information; Normal and Abnormal (Adventitious) Normal sound is the respiratory sounds of healthy subjects and can be described as; open airway, normal respiration rate \& rhythm and chest expansion and relation that occur in a standard way. In a word, that is the result of the normal functioning of the lung. The meaning, do not involve any kind of lung disease. On the other hand, by the effect of the changes in the structure of the lung, the characterization of the sounds has been changed. This change is to hear the "extra or additional" sounds so unusual except the normal sounds. Those kinds of sounds refer to adventitious or pathological respiratory sounds. These sounds generally an indicator of impending abnormality in the lungs such as an obstruction in the airway passages or pulmonary disease or deformation of the respiratory system by cause of any reason. This is a great importance in terms of diagnosis of the disease. Two main group of adventitious sounds are called: continuous (wheeze, rhonchi, and stridor) and discontinuous (crackles, fine crackles, and coarse crackles). Generally, discontinuous sounds are short duration and sporadic, in contrast, continuous sounds are a long duration and may see full ventilation cycle on the subject.

Auscultation is a process of listening body sounds for the purpose of the health condition of the respiratory system. Doctor/physician performs auscultation by using a stethoscope. It is used to transform body sounds from the body surface to the human ear. It has some adventitious like its simplicity, easy to use and generally cheap on the other hand it has some limitations like subjectivity, clinical experience of a physician, listening skills and as much knowledge about lung sounds structure.

In recent years with the advance of computational methods, many researchers and investigator group have described different types of approach for analyzing and classification of lung sounds. It offers a perfect advantage to analyze, recognize and classification of respiratory sounds. Additionally, and most importantly in this way, the effect of subjective factors will be minimized or removed. In general term, computerized lung sounds analysis consist of three particular and essential process or step: pre-processing, feature extraction-selection and lastly decision-making stage. Pre-processing, generally performed for noise or artifact reduction and enhance the quality of the sounds for other steps most importantly for feature extraction part. Feature extraction process converts raw data some type of parametric, special features. To find out smallest, shortest and the best complete dataset, from longest dimensional dataset. Lastly, decision-making process categorizes and analysis of lung sounds data according to health condition. Up to this time, various studies have been made using different feature extraction -selection and classification algorithms. The studies related to this subject are as follows:

Dokur and Olmez., [1] classified respiratory sounds as healthy and patients with asthma by using the classification methods as "Grow and Learn" Neural Network (GAL), Kohonen Network and Multi-Layer Perceptron (MLP). Grow and Learn Neural Network classification performance was reported $98 \%$ for both cases in asthma and normal subjects. Kandaswamy et al., [2] categorized lung sounds like normal, wheeze, crackle, squawk, stridor, or rhonchus. Discrete Wavelet Transform (DWT) level of seven was applied for the feature extraction method of lung sounds signal. ANN classification accuracy was reported $90 \%$ for categorization of lung sounds. Abbasi et al., [3] classified normal and abnormal lung sounds by using NN and Support Vector Machine (SVM) based on Wavelet coefficients. Classification result indicates that SMV is successfully classified with an accuracy of $93.51-100 \%$ for the classification of lung sounds. Uysal et al., [4] presented a classification of normal and abnormal lung sounds by using Wavelet coefficient intended. 34 recording lung sounds were taken from R.A.L.E database 14 normal, and rest of them abnormal. Feature vectors were obtained by using wavelet 
coefficients and were determined by using Daubechies-7. In the classification process, ANN and SMV were respectively used. The results of the study show that both classifiers give $100 \%$ performance for the classification of lung sounds.

In this study, the frequency spectrum of normal and asthmatic patients is obtained by using Fast Fourier Transform (FFT) after dividing recorded sounds from the right and left parts of the lung into inspiration and expiration phases. The most important advantages extracted respiratory cycle are;

1)the size of sound data will be less and hence the classifier process becomes faster and more accurate.

2)inspiration and expiration phases may have distinctive characteristics of sounds.

One of the most important goals of this work, use the full cycle of each phase, not only one cycle of inspiration or expiration phases. In each group has at least 4 cycles of data. Depending on different sounds, the correctness of lung disease decision ratio will increase due to the presence of abnormal sounds either inspiration or expiration. Proposed cascade classifier methods are combined of two subsystems: the first one is constituted by using unsupervised fuzzy clustering technique to choose the best patterns that ideally shows its class attributes in date set. The second is realized the classification of extracted features for each pattern by using different supervised classification algorithms such as ANN, Naive Bayes and SVM.

The rest of the article organized as follows, in section 2 gives a point of view about the analysis process of the recorded lung sounds, signal acquisition and filtering information. Section 3 describes theoretical background information about classification algorithms. In section 4, this is the main part of the article and included in the classification results according to health condition of lung sounds and finally summarization the paper.

\section{METHOD}

In this section, we will first get information about how lung sounds are collected and database information. Then, feature extraction and classification algorithms used are given. The flow diagram of this section is given below (see Figure 2.1)

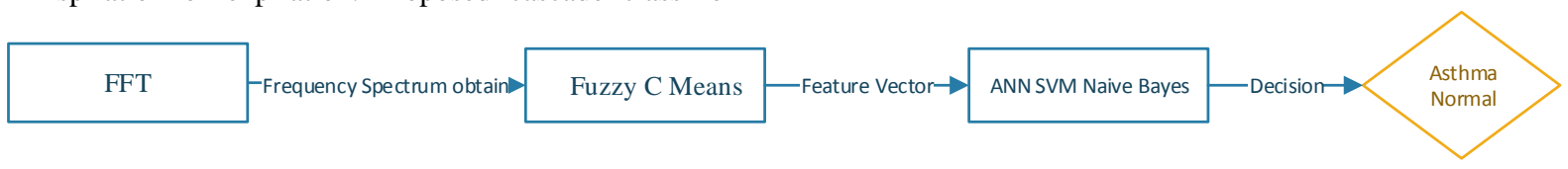

Fig 2.1: Decision making stage

\subsection{Lung Sounds Signal Acquisition \& Pre- Filtering}

In this study, ECM T-150 Sony electrets condenser type microphone with a sampling frequency $8 \mathrm{kHz}$ and 16-bit resolution used to get a signal over the basic location of the chest wall. The microphone impedance is $2.2 \mathrm{k}$ and response bandwidth in between $50-15000 \mathrm{~Hz}$. The microphone used one direction for sounds recording by stethoscope head. These ranges of bandwidth include not only lung sounds but also, heart muscle and other sounds which come from other intracorporeal sounds. Therefore, the sounds have to be filtered before analyzing the signal. Figure 2.2. shows the basic outline of the system used this study.

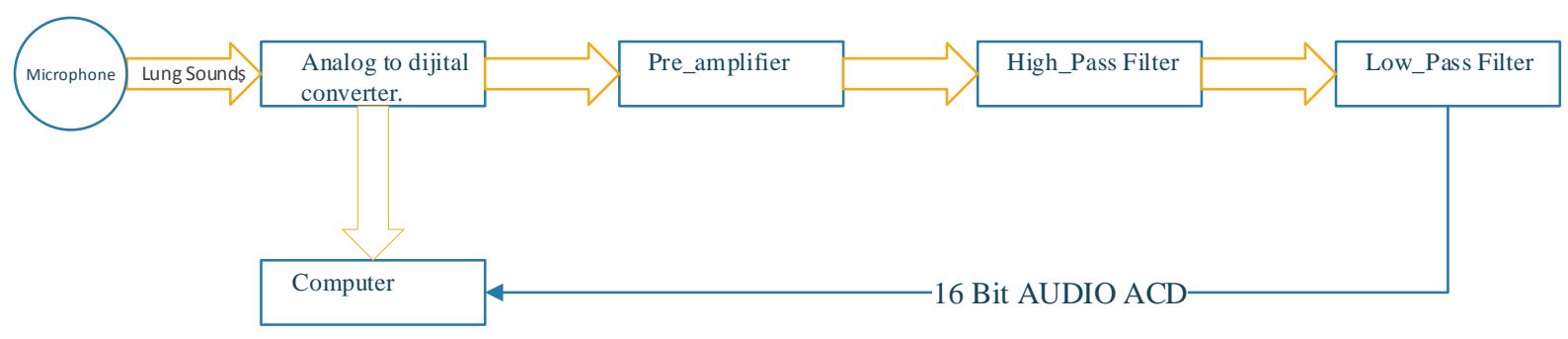

Fig 2.2: Applied System Block Diagram

Filtering stage divided into two parts. In the first stage, Bessel type $5 \mathrm{~Hz}$ high pass filter with analog filter order of six was used and we utilized OP275 operational amplifier to build the circuitry of the first stage. In the second stage, however, Butterworth type low pass filter implemented as a switched capacitor filter order eight was used and the MAX295 integrated circuit was employed to build the circuitry of the second stage. When recording respiratory sounds, the common problem is that sounds are not fully noticeable because of the combination of a heartbeat, muscle movement and other sounds which come from work of other organs in the human body. For that reason after removing DC components, to filter these undesired and nonessential sounds, band-pass finite impulse response filter (FIR) the range of 100 $\mathrm{Hz}$ to $2000 \mathrm{~Hz}$ applied recorded lung sounds signals. Figure 2.3 and Figure 2.4 illustrates filtering stage for healthy and asthmatic lung sounds original input signal and after FIR filtering stage. All the lung sounds are recorded during normal breathing in regular respiration cycle, both left and right posterior bases of the lung in the same location: positions P4 and P5, respectively suggested by the physician. Duration of each sounds in between 10-14 seconds. Lung sounds recorded College of Medicine at University of Gaziantep hospital from volunteers who visit lung disease clinic under the control of pulmonary physician. Distribution of the age in between 1955 in both normal and asthma patients. In this research 11 normal and 22 asthmatic sound signals are used.

Each lung sound signals consists of at least 4 inspiration and expiration respiratory cycle with a sampling frequency 8000 $\mathrm{Hz}$ and 16 bits. 

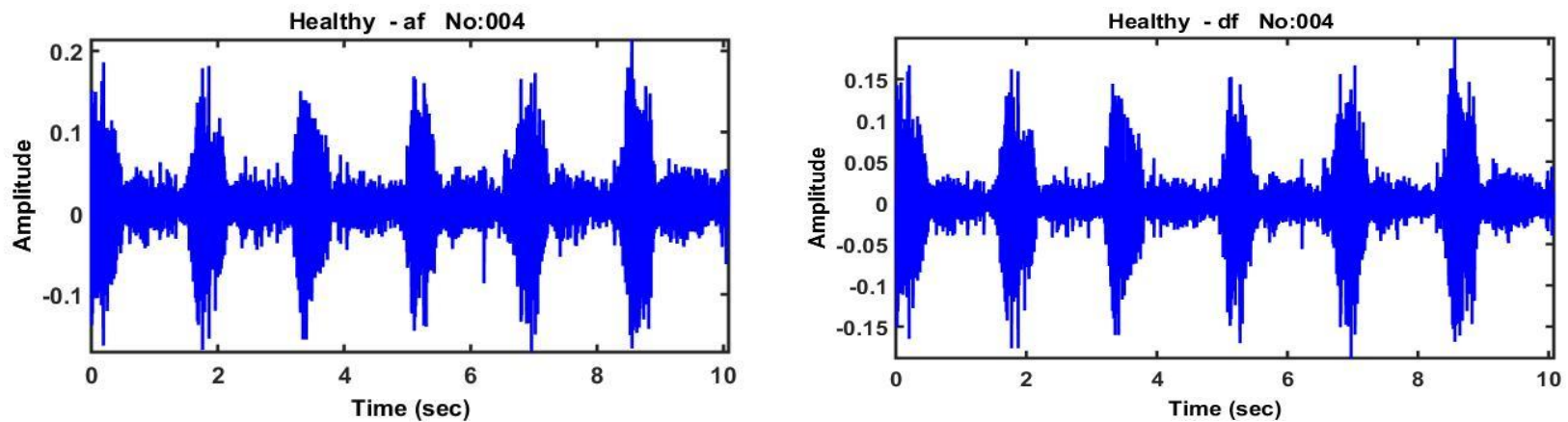

Fig 2.3: Original waveform of the input signal healthy subject and after filtering.
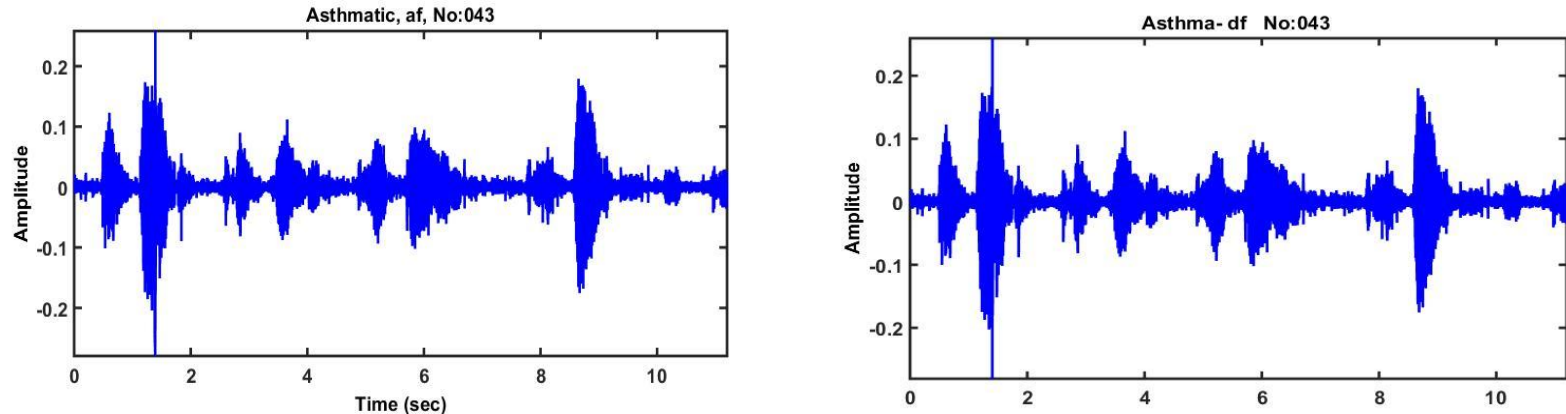

Fig 2.4: Original waveform of the input signal asthma patient and after filtering.

Pay attention to Figure 2.3, in health or normal lung sounds breathing and breathing pattern periods are certain. But in Figure 2.4, asthmatic lung sound breathing pattern, ventilation cycle is different than normal lung sounds. In general, asthmatic patients breathing pattern inspiration or expiration or in both cases deteriorating changes occur.
The frequency spectrum of normal and asthma patient is obtained by using FFT. The frequency spectrum for normal and asthmatic lung sounds ventilation cycle inspiration \& expiration phases information figure out in the following figures (Figure 2.5 and Figure 2.6).
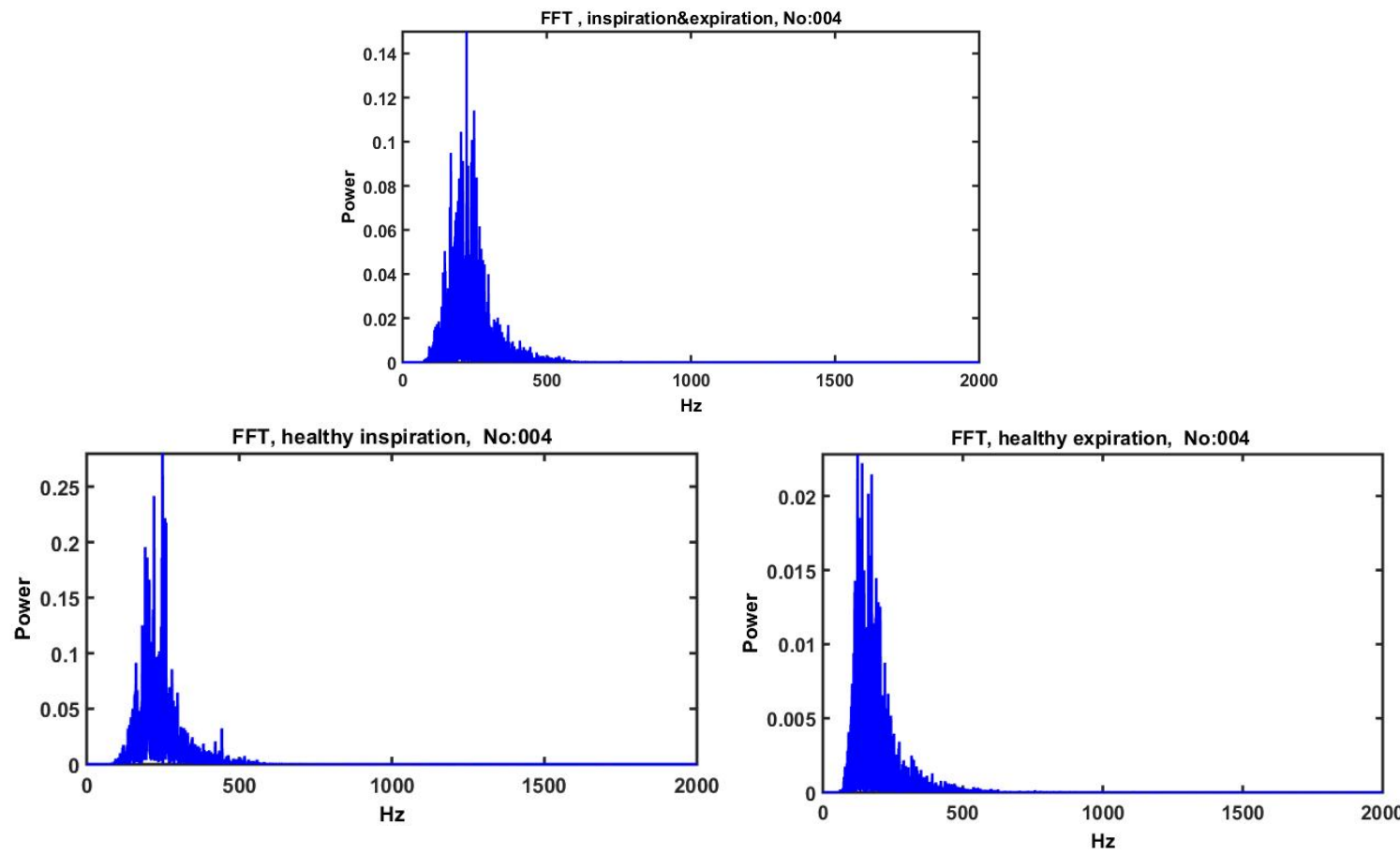

Fig. 2.5: The frequency spectrum of healthy lung sounds; full cycle, inspiration, and expiration. 

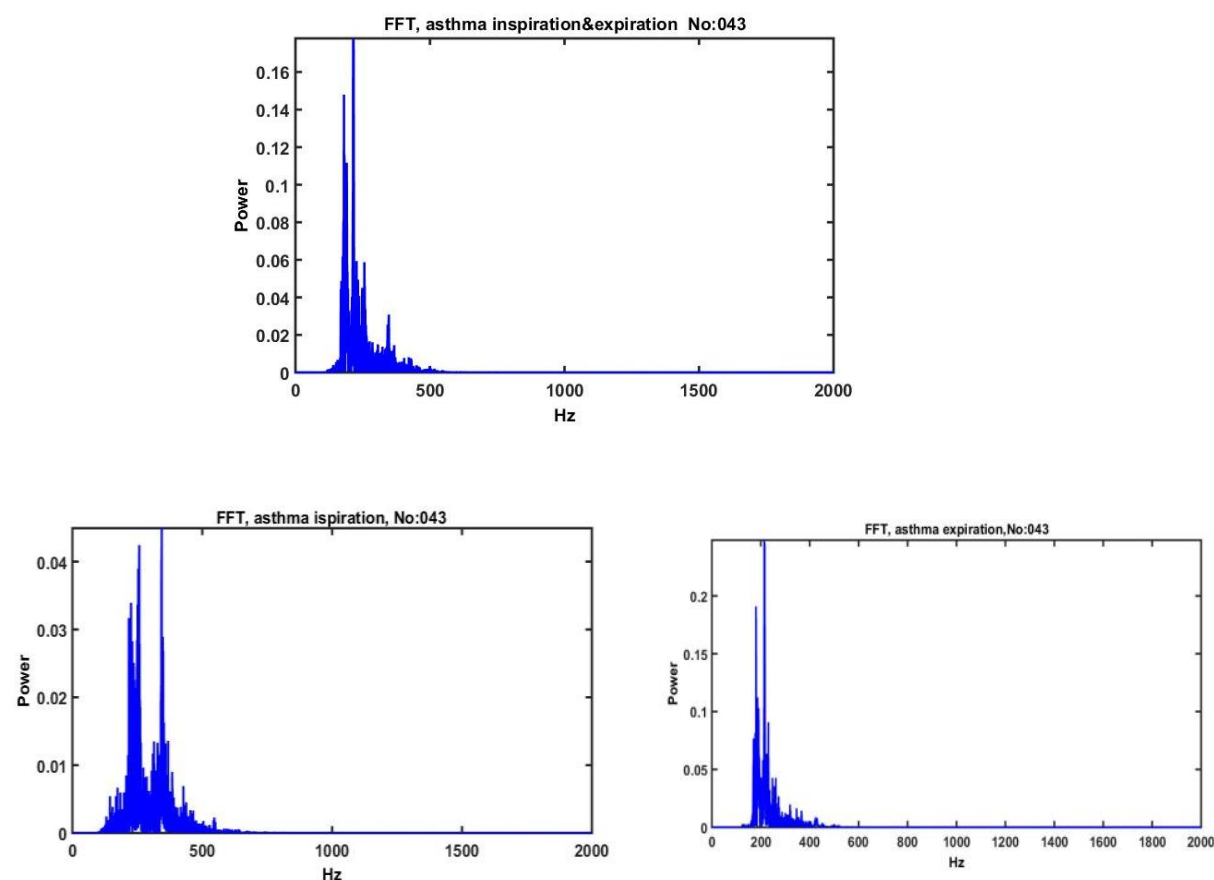

Fig. 2.6: The frequency spectrum of asthmatic lung sounds; full cycle, inspiration, and expiration.

\subsection{Background Information}

\subsection{Unsupervised Fuzzy C Means \\ Clustering and Implementation}

Fuzzy c-means is an unsupervised algorithm for clustering, that allows one part of the data related to two or more cluster. Based on the iterative procedure, Fuzzy c-mean is minimized an objective function, which defines the distance from any data point to cluster center weight by that point membership grade. Calculation of fuzzy membership in Fuzzy c-mean based on the computation of relative distance. FCM techniques widen this concept to cooperator of each pattern with every cluster by using a fuzzy membership function, resulting in a clustering and not in a partition center. The Fuzzy c- mean technique minimizes the within-class sum square error under the following condition [5] . Where $\mathrm{N}$ shows the number of data, $\mathrm{c}$ is

$$
\begin{aligned}
& \sum_{j=1}^{c} m_{i j}=1 \quad \mathrm{i}=1,2,3 \ldots \ldots, \mathrm{N} \\
& \sum_{\mathrm{j}=1}^{\mathrm{N}} \mathrm{m}_{\mathrm{ij}}>0 \quad \mathrm{i}=1,2,3 \ldots \ldots, \mathrm{N}
\end{aligned}
$$

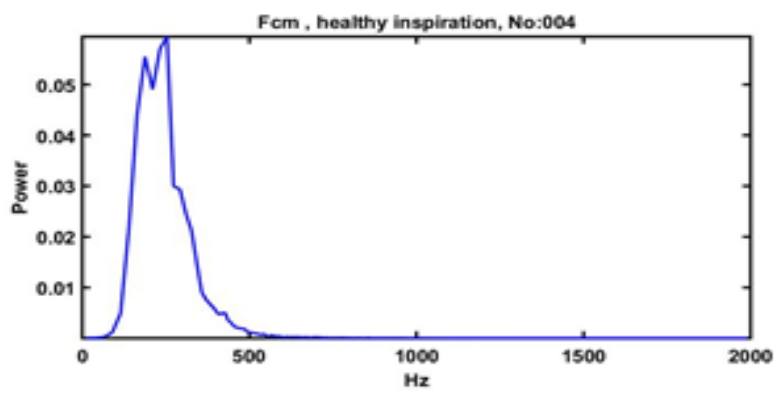

$$
\begin{aligned}
& \mathrm{G}=\sum_{\mathrm{i}=1}^{\mathrm{N}} \sum_{\mathrm{J}=1}^{\mathrm{c}} \mathrm{m}_{\mathrm{ij}}^{\theta} \mathrm{d}^{2}\left(\mathrm{x}_{\mathrm{i}}, \mathrm{c}_{\mathrm{i}}\right) \\
& m_{i j}=\frac{d_{i j}^{2 /(\theta-1)}}{\sum_{j=1}^{c} d_{i j}^{2 /(\theta-1)}}=c_{j} \frac{\sum_{i=1}^{N} m_{i j}^{\theta} x_{i}}{\sum_{i=1}^{N} m_{i j}} \\
& \mathrm{i}=1,2,3 \ldots \ldots, \mathrm{N} \quad \mathrm{j}=1,2,3 \ldots \ldots, \mathrm{c}
\end{aligned}
$$

defined the number of classes $(1<\mathrm{c}<N), c_{j}$ is the vector describing the center of class $\mathrm{j}, x_{i}$ is the vector describing singular data $i, d^{2}\left(x_{i}, c_{j}\right)$ (or $d_{i j}$ ) is the squared distance (like Euclidian distance) $x_{i}$ and $\mathrm{c}_{\mathrm{j}}$ considering a selected description of distance, and $\phi$ is the fuzzy exponent that interval from $(1$, $\infty)$ and defines the degree of fuzziness of the last solution. With $\theta=1$, the degree of fuzziness of the last solution. With $\theta=1$, the solution is a hard partition. As infinity approximations, the solution approaches its highest degree of fuzziness. The minimization of the objective function $G$ (Eq.3) finds the solution for the membership function and the cluster center. Fuzzy c means findings are detailed in Figure 2.7 and Figure 2.8 .

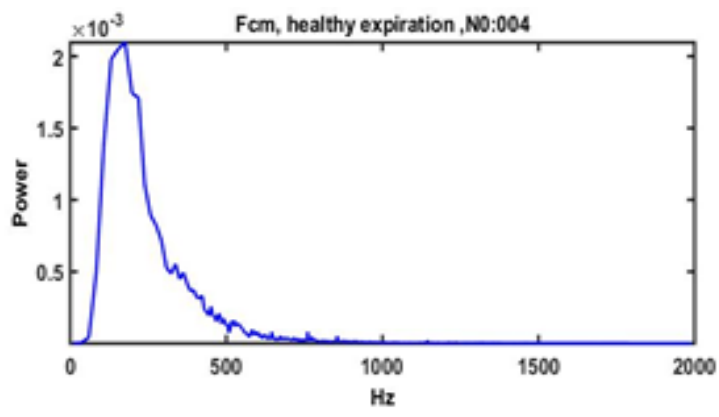

Fig. 2.7: FCM application of Figure 2.5 

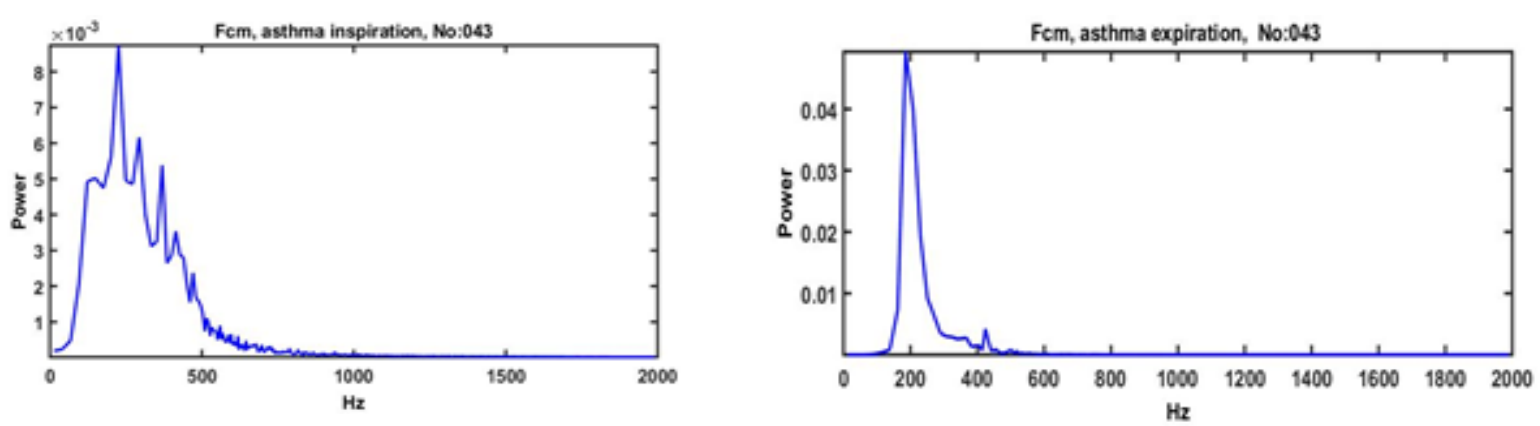

Fig. 2.8: FCM application of Figure 2.6

\subsection{Supervised Machine Learning Algorithms}

Machine learning is about learning to predict from samples of target behaviors or past observations of data. Machine learning algorithms are divided as supervised and unsupervised learning simply. Supervised learning, where the algorithm creates a function that maps inputs to target outputs. The learner then compares its actual response to the target and adjusts its internal memory in such a way that it is more likely to produce the appropriate response the next time it receives the same input. Unsupervised learning methods are a set of inputs. There are no target outputs (no any desired or target examples). The learner receives no feedback from the environment and uses the competition strategy. In this study, well known and efficient supervised machine learning algorithms such as ANN, Naive Bayes, SVM which are used to classify normal and asthmatic sounds.

\subsubsection{Artificial Neural Network (ANN)}

ANN, inspired by the human brain developed, through main links connecting to each other and each of them has its own memory that is information processing elements. ANN are modeled by inspired of biological neural networks but has a simpler structure than them. ANN system modeling is used in classification and interpretation works such as recognition and interpretation of speech recognition, handwriting recognition, fingerprint recognition, physiological signs (heart function, brain function, respiratory function, etc.) In addition to this, ANN is also used to solve complex problems such as business, industry, finance, industry, education and science fields. In this study, one of the most important and widely used models of ANN, that is, Multi-Layered Perceptron (MLP) architecture, supervised error Back-Propagation learning algorithm has been used. In all layers, sigmoid activation function is used to obtain best results. In this study, the learning rate: 0.95 and for momentum coefficient: 0.95 as the best constants were found to be.

A number of nodes in the input layer are the same number of the feature vector, multi-layered of ANN are 500, 70 nodes in the hidden layer, and finally in the output layer, including 2 , as asthma and normal. The number of nodes in the hidden layer is determined according to changeable results of the node count of the Error Rate which has made in Training (including training) and testing (testing) stages. 50\% of the data which is given for classification was used for test and the other half is for learning.

\subsubsection{Support Vector Machine (SVM)}

SVM is specifically used to solve both linear and nonlinear classification problem in a supervised manner which presents one of the kernel-based methods. SVM is used for classification of both linearly separable and inseparable data. SVM is to form an optimal separating hyperplane in such a way that the margin of separation between two classes is maximized [6]. This approach can be extended to patterns which are linearly separable by transformations of original data to map into new space due to using kernel trick. SVM assigns support vectors that are formed a margin between two classes, thus providing that the data is more separable than in the case of the other classifiers. Support vector machines are naturally resistant to overfitting because any interior points aren't going to affect the boundary.

\subsubsection{Nä̈ve Bayes}

Naïve Bayes classifier is an easy but effective probabilistic classifier based upon Bayes' theorem with strong (naive) independence assumptions. Naïve Bayesian classifiers assume attributes which have independent distributions. Naïve Bayes classifier is trained by a supervised learning setting which uses the maximum likelihood algorithm for parameter estimation [7]. The derivation of maximumlikelihood predicts for the Naïve Bayes method, in the easy case where the underlying classes (or labels) are found in the training data. A Naïve Bayes Classifier is a program that estimates a class value given a set of set of attributes. For each known class value;

- Compute probabilities for each attribute, conditional on the class (or label) value,

- Use the product rule to obtain a joint conditional probability for the attributes,

- Use Bayes rule to derive conditional probabilities for the class (or label) variable.

Advantages and disadvantages of Naïve Bayes classifier can be summarized as:

$>$ Advantages:

- Fast to train and fast to classify

- Not sensitive to unrelated features

- Operates real and discrete data

- Operates streaming data well

$>$ Disadvantages:

Assumes independence of features

\section{FUZZY CLUSTERING -BASED CASCADE CLASSIFIER}

Fuzzy clustering-based cascade classifier is a hybrid learning algorithm which integrates both unsupervised Fuzzy c-mean clustering and supervised classifiers such as ANN, SVM, and Naïve Bayes. This method is defined and 
used by [8]-[10]. As seen in Figure 3.1, the structure of the proposed Fuzzy clustering-based cascade classifier which consists of two phases. At the first phase, inputs and outputs values of the feed-forward neural network are found out by using Fuzzy c-mean clustering algorithm. At the second phase, these clustering data is applied as inputs with target values of classifiers which have supervised learning. The number of data set is decreased by using Fuzzy c-mean clustering before inputs are applied to the classifier.

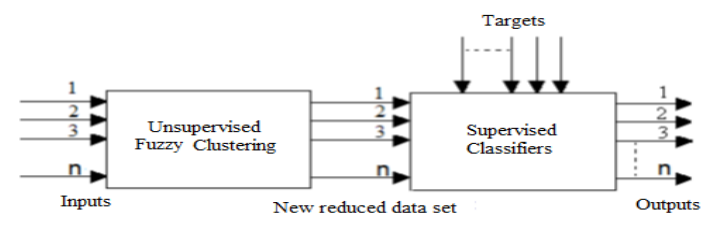

Fig. 3.1. Structure of Fuzzy Clustering Based Cascade Classifier

\section{RESULT}

This study has proposed the performance of different fuzzy clustering-based cascade classifier ANN, SVM, and Naïve Bayes for classification of respiratory sounds as normal or asthmatic based on ventilation cycle. Firstly, the frequency

Table 1: Classification accuracies of normal and asthmatic classes using ANN, SVM, and Naive Bayes classification algorithms

\begin{tabular}{|c|c|c|c|}
\hline Lung sounds & ANN & SVD & Naïve Bayes \\
\hline Inhalation & 88.00 & 84.6 & 94 \\
\hline Exhalation & 71.00 & 96.7 & 90.9 \\
\hline
\end{tabular}

Table 2: Error rates and class precision based on confusion matrix where (TP: true positive, FP: false positive, FN: false negative, TN: true negative, P: class precision, ER: error rates)

\begin{tabular}{|c|c|c|c|c|c|c|c|}
\hline Method & Phase & $\mathrm{TP}$ & FP & FN & $\mathrm{TN}$ & $\mathrm{P}$ & ER \\
\hline \multirow[b]{2}{*}{ 忞 } & Inhalation & 9 & 1 & 1 & 21 & $88 \%$ & 0.1 \\
\hline & Exhalation & 8 & 3 & 5 & 17 & $71 \%$ & 0.6 \\
\hline \multirow{2}{*}{$\sum_{3}^{2}$} & Inhalation & 8 & 2 & 3 & 20 & $84.6 \%$ & 0.1 \\
\hline & Exhalation & 10 & 0 & 1 & 22 & $96.7 \%$ & 0.03 \\
\hline \multirow{2}{*}{ 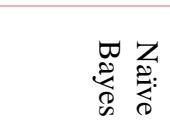 } & Inhalation & 10 & 1 & 1 & 21 & $93.2 \%$ & 0.02 \\
\hline & Exhalation & 10 & 2 & 1 & 20 & $90.9 \%$ & 0.03 \\
\hline
\end{tabular}

\section{REFERENCES}

[1] D. Z. and O. T., "Classification of respiratory sounds by using an artificial neural network," Int. J. Pattern Recognit. Artif. Intell., vol. 17, pp. 567-580, 2003.

[2] A. Kandaswamy, C. S. Kumar, R. P. Ramanathan, S. Jayaraman, and N. Malmurugan, "Neural classification of lung sounds using wavelet coefficients," Comput. Biol. Med., vol. 34, pp. 523-537, 2004.

[3] S. Abbasi, R. Derakhshanfar, A. Abbasi, and Y. Sarbaz, "Classification of normal and abnormal lung sounds spectrum of normal and asthmatic sounds full cycle both inspiration-expiration were obtained from [11] row data. Then this obtaining data of the respiratory sounds were extracted by using the Fuzzy c-mean clustering. Respiratory sounds ventilation cycle (inhale \& exhale) characteristic information is to provide essential information about respiratory disorders and pathological condition of patient. Because, inhalation and exhalation phases have their noticeable characteristics as mentioned related to different pathological information so this information is to comprise very important phenomena in the diagnosis of respiratory disease and decision-making about the health condition of the subject. Classification accuracies and detail information of different machine learning algorithms performance shown in Table 1 and Table 2. Based on the ventilation cycle, in the inhalation process Naïve Bayes gave better results \%94, and SVM $\% 96.7$ for exhalation process. In the whole process of classification performance based on breathing pattern exhalation cycle gave remarkable results than inhalation cycle.

In clinical cases based on structure of lung, when inhalation occurs airways widen but in exhalation, they get narrower and more information about the disease is obtained. 
[6] B. Karlik, "Machine learning algorithms for chacarterization of EMG signals," Int. J. Inf. Electron. Eng., vol. 4, pp. 189-194, 2014.

[7] B. Karlik, "Hepatitis Disease Diagnosis Using Backpropagation and the Naive Bayes Classifiers," J. Sci. Technol., vol. 1, pp. 49-62, 2011.

[8] B. Karlik, M. O. Tokhi, and M. Alci, "A Fuzzy Clustering Neural Network Architecture for Multifunction Upper-Limb Prosthesis," IEEE Trans. Biomed. Eng., vol. 50, no. 11, pp. 1255-1261, 2003.

[9] B. Karlık, "The effects of fuzzy clustering on the backpropagation algorithm," in International Conference, 2014
[10] B. Karlık and K. Yüksek, "Fuzzy Clustering Neural Networks for Real-Time Odor Recognition System," J. Autom. Methods Manag. Chem., vol. 2007, p. 38405, 2007

[11] Karlık Bekir, Güçlü, Güneş, Öz Halil Rıdvan, "Respiratory Sounds Classification Fuzzy C-means , Principal Component Analysis and Artificial Neural Network," in 1st International symposium on computing in science \& engineering Bioengineering congress, 2010. 